\title{
Ownership Identification of Reindeer Calf Using Wireless Sensor Networks (WSN)
}

\author{
Mohamad Y. Mustafa \\ Department of Technology, Narvik University College, 8505 Narvik, Norway
}

\section{Email address:}

myfm@hin.no

\section{To cite this article:}

Mohamad Y. Mustafa. Ownership Identification of Reindeer Calf Identification Using Wireless Sensor Networks (WSN). Communications. Vol. 3, No. 3, 2015, pp. 56-62. doi: 10.11648/j.com.20150303.11

\begin{abstract}
This paper presents a technique for identifying the ownership of new-born reindeer claves using wireless sensor networks (WSNs), which is an important tool that can be used to acquire useful information about animals' activities and movements. Reindeer are semi wild and they give birth while in the wild. Although reindeer cows usually carry identification tags or signs of their owners, it is difficult to identify the ownership of the calves within a mixed herd. Currently, identification is performed in the traditional way which is stressful on both animals and herders and quite costly and time consuming. Various potential WSN technologies are considered in this work with special attention paid to RFID tags and subsequently, a method based on Wi-Fi enabled RFID tags is proposed. Localization techniques play an important role in the proposed method, hence localization algorithms are discussed thoroughly. This work aims to pave the way for the use of wireless sensor networks for the purpose of matching mother reindeer to their calves and for other matching purposes in animal welfare and industry.
\end{abstract}

Keywords: Wireless Sensor Networks (WSN), Reindeer Herding, RFID Tag, Distance Estimation, Localization, Animal Tracking, Wi-Fi-enabled RFID

\section{Introduction}

Reindeer herding is the major economic activity of the Sámi people, who are the indigenous people of northern Sweden, Norway and Finland. The Sámi people lived and worked in reindeer herding groups, which consisted of different families within a designated area and which were formed of working partnerships, where members had individual rights to resources, but helped each other with the management of the herds, or when hunting and fishing. Reindeer husbandry in Norway today is a small industry on a national scale, but is important economically and in employment terms, it is also one of the most important parts of the Sámi culture.

Reindeer herding in Norway was regulated in 2007 and allowed only those who have the right to a reindeer earmark to undertake reindeer husbandry in the Sámi reindeer herding area. This condition applied only to persons who are Sámi themselves, whose parents or their grandparents have or had reindeer herding as their primary occupation.

A reindeer earmark is a combination of one to many cuts in a reindeer's ears, which all together tell who the reindeer owner is. There are around 20 different approved cuts in addition to some 30 different combinations of cuts, which have their own names. A committee is in charge of approving earmarks before they are implemented and all reindeer in the Sámi reindeer husbandry area shall be marked with the owner's registered earmark by 31 October the same year as it is born.

Reindeer are semi-wild and require large areas for their grazing, they are also often frightened and are forced to flee from natural pastures. Most of the time, they are left to wander freely unaccompanied with their herders [1].

Reindeer herders typically make two migrations with their animals each year. During winter, reindeer are left to breed in the highlands, but just before spring, when food becomes scarcer in the mountains, natural instinct directs the herd to migrate to the low grounds for greener pastures [2]. Reindeer are intercepted by their herders and are taken to the spring pastures before the snow melts and before the mothers start calving. In spring, they move back to the mountainous coastal region where they feed throughout the summer, the calves are soon born and stay with their mothers for protection [3].

In autumn, the herders return to the grazing locations to gather the reindeer from the mountains before the breeding season starts. This is the time when the new calves are 
branded. Each calf is captured, vaccinated and is given an identification tag or ear mark to distinguish which cow the calf belongs to and to which owner they belong [2].

As meat production became more important since the beginning of the $20^{\text {th }}$ century, reindeer herding became more extensive and Sámi reindeer herders started to implement modern technologies, such as snow mobiles and various other mechanical and electronic aids, which became a major feature of modern reindeer herding. Amongst those technologies, ear identification tags are being used extensively. However, identifying the ownership of new-born calves remains a major problem for reindeer herders [1].

Recognising and matching reindeer calves to the different mothers is traditionally performed in two steps; first, the animals are gathered in a small pen. All female reindeer get individual numbers sprayed on the skin at each side of the animal. Each owner uses one specific colour. Unique number plates are hanged to collars around the necks of the calves. Thereafter, the animals are released to larger pens to calm down and are put under surveillance by reindeer owners and herders using binoculars, in order to identify which reindeer calf is following which cow. Thereafter, reindeer owners compare the different observations to guaranty that the correct mother is matched to her calf [4].

The next step is to gather the reindeer herd again in a small pen, capture the calves, remove the number plates and replace them with the approved ear cutting or a tag. This handling procedure is a painstaking process, which demands considerable amount of man power, and long periods of observation of the herd, and is stressful to both herders and animals alike [4].

With the advancement of wireless communications and the wide spread of wireless networks, it has become possible to utilise those technologies for the benefits of reindeer herders and their animals. This paper proposes a technique based on WSN technology for ownership identification of new-born reindeer calves. Various prospective identification technologies such as RFID tag, GPRS collars or UHF nodes and gateways are discussed in this work to explore their feasibility for the proposed application [1].

In this concept, it is proposed to equip the reindeer cows and calves with transmitter devices of the selected technology, and monitor the movement of the herd in a confined space, facilitated with a grid of signal receivers, corresponding to the transmitter device attached to the reindeer. A suitable algorithm has to be devised to analyse the gathered data and recognise the pairs of tags which keep close together most of the time, which are reckoned to be the mother reindeer and her calf.

Due to the fact that reindeer are semi wild, they tend to be more cautious as opposed to domestic animals. This makes it difficult to mount receivers too close to the animals, which makes the use of passive RFID tags not feasible. Hence, it is imperative to use either active RFID tags, which operate at higher frequencies than passive tags because of their onboard power source and can communicate with their respective receivers across 20 to 100 meters, and can track the movement of highly mobile objects in wider areas than passive tags, or use GPRS collars or UHF nodes, which are capable of transmitting signals to long distances, but at a higher cost [5].

It is aimed here to present a new method for reindeer identification based on electronic communications techniques. The requirements of the WSN system with relation to animal behaviour and animal welfare will be discussed. Various potential WSN technologies and localization algorithms will be discussed to define system requirements, and to propose the most appropriate sensor network technology and localization technique with adequate justification. It is claimed that the proposed technique offers a modern approach, and a more cost effective and less stressful solution to the problem of identification of reindeercalf ownership, and can be used for other applications under similar conditions.

\section{Proposed Concept of Identification}

A technique based on wireless sensor networks (WSN) to identify the ownership of the calves is presented in this work. It is proposed to furnish the new born calves with an electronic identification device, such as RFID tags, GPRS collars or UHF nodes, with a system set-up facilitated with receivers, gateways and necessary software. The reindeer herd is confined in a restricted space, which is equipped with a grid of signal receivers, corresponding to the transmitter devices attached to the reindeer, and using proper localization algorithms. Movement of transmitter tags is tracked in order to recognise the pairs of tags which keep close together most of the time, which indicate the mother and calf reindeer.

Due to the fact that reindeer is a wild animal species, they tend to be more cautious than domestic animals; hence they tend to avoid confined spaces and close objects mounted by humans, which makes the use of passive RFIDs less efficient due to their low range, as the receivers have to be placed at a close proximity to the RFIDs. This demands the application of active RFIDs to both mothers and calves, or the use of other sensor network devices such as GPRS collars or UHF nodes and respective gateways. For example, active RFID tags operate at higher frequencies than passive tags because of their on-board power source, commonly $455 \mathrm{MHz}, 2.45$ $\mathrm{GHz}$, or $5.8 \mathrm{GHz}$ - depending on application's read range and memory requirements. Readers can communicate with active RFID tags across 20 to 100 meters. In the same way, tags can track the movement of highly mobile objects in wider areas than passive tags [5].

In this procedure, the pen where the animals are to be gathered for tagging is facilitated with a Wi-Fi network, assuming that the Wi-Fi network is readily available, but this will eventually be the choice of this study as will be justified in due course. Wi-Fi routers are mounted on posts at the centres of the circles indicated in figure 1 , which indicate the coverage range of the routers. 


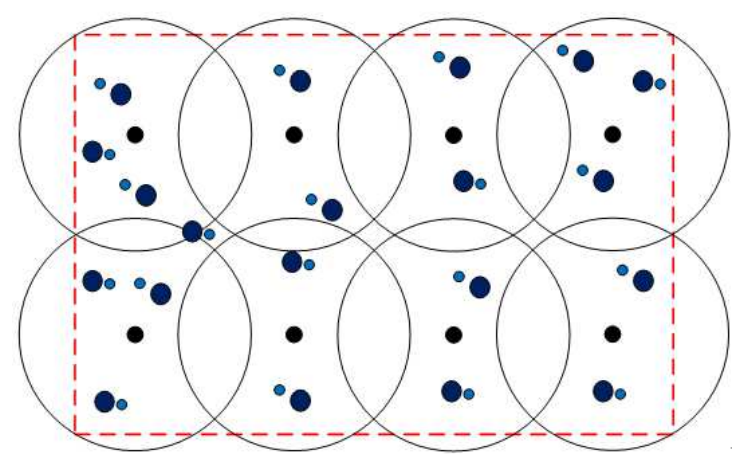

Figure 1. Proposed configuration of the localization set-up. Suggested area of the pen is $40 \times 80 \mathrm{~m}$ (dashed rectangle). Large circles represent coverage area of the gateways, routers are represented by the black circles at the centres of the larger circles. Mother and calf reindeer are represented by the large and small pairs of coloured circles. Schematic represents possible configuration of the Wi-Fi coverage.

Coverage of the wireless network is configured in such a way to ensure exposure of the whole pen, with minor inevitable pockets of uncovered areas, which have to be kept to a minimum. RFID tags are tracked and location data is logged into a computer programme, where data is analysed to match couples of tags together.

\section{Potential Tracking Technologies}

There are many examples in the open literature on the application of WSN for animal welfare. A WSN for wildlife monitoring was implemented by Vishwas et al. [6]. In their system, they designed neck collars with sensor nodes to collect data about the desired parameters from the vicinity of the animal and transmit it to the base station. This information was used by wildlife scientists for habitat monitoring and behavioural habits of the animals under observation.

A wireless communication solution that fulfils the requirement for intensive condition monitoring of individual animals, aggregation and timely reporting of data to the farm manager designed for both loose house dairy cattle and free ranging beef cattle was offered by Kwong et al. [7]. Lowcost, low power-consumption sensor nodes are utilised in the proposed method as the base elements of a data gathering and communication infrastructure. Performance of the platform communication protocol was evaluated through farm trials and laboratory experiments. Experimental results indicated that the system performed efficiently while conforming to the limitations associated with WSN implementations.

A number of potential WSN technologies for animal tracking will be considered in the following sections. Greater attention will be given to RFID technology, as it is widely used in animal identification. GPS and Wi-Fi technologies will also be considered being widely available at affordable prices.

\subsection{Radio Frequency IDentification (RFID)}

RFID is a low-cost technology, which is widely used for electronic identification and tracking. RFID offers substantial advantages for businesses, allowing automatic inventory and tracking on the supply chain. This technology plays a key role in pervasive networks and services [8]. RFID enables real-time identification of devices and users through remote storage and retrieval of data. This data can also be linked to location, which optimizes the usage of RFID [9].

RFID networks usually comprise three different units; tags, readers, and servers, as shown in Figure 2. Communication between RFID tags and the readers is achieved through radio frequency energy. However, tags are powered in different methods. An active tag and its RF communication circuitry are constantly powered by an internal battery. Readers can thus transmit very low-level signals, and the tag can reply with high-level signals [4]. Additional functionalities such as extended memory or sensors can be embedded within an active tag. On the other hand, passive tags are not equipped with internal power supply, nevertheless, they automatically reflect the carrier signal received from a reader. The size of passive tags is smaller and are cheaper than active tags, but their functionalities are very limited.

A third type of RFID tags is the semi-passive tags. These tags communicate with the readers like passive tags, but they embed an internal battery that constantly powers their internal circuitry [9].

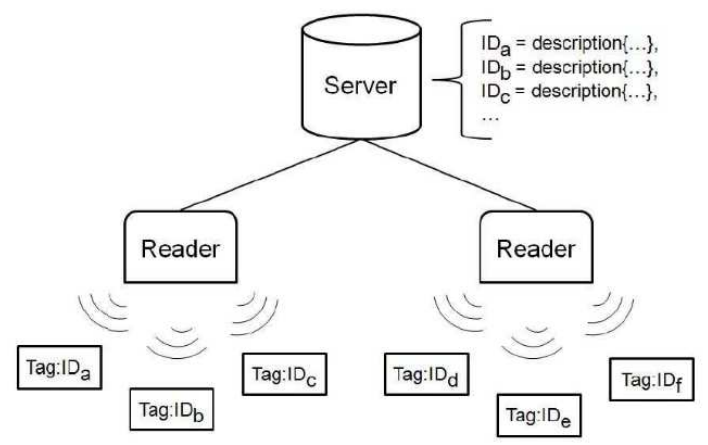

Figure 2. Classic architecture of RFID system [9].

RFID reader systems comprise three parts. The first one is an RF interface that communicates with the tags in their read range in order to retrieve tags' identities. The second one is a communication interface, generally IEEE 802.11 or 802.3 , for communicating with the servers. The third part constitutes one or several servers, which collect tags' identities sent by the reader and perform specific calculations such as the position of the tag through a localization method. The servers can be interconnected between each other, and embed the major part of the middleware system.

Usage of RFID systems can be classified in two main categories [10]:

1. Monitoring and authorizing, example applications are livestock or people equipped with RFID tags.

2. Systems where RFID tags are not permanently attached to entities, such as authorizing systems in building access controls, where tags are embedded inside cards 
or keys.

\subsection{Global Positioning System (GPS)}

The development of universal positioning solutions was promoted by GPS. This is due to the fact that GPS can meet the majority of attributes of ubiquitous positioning systems, such as accuracy, availability and reliability under ideal operational conditions. However, these attributes can deteriorate to unacceptable levels under certain environments, with often only weak GPS signals available [11].

\section{3. $\mathrm{Wi}-\mathrm{Fi}$}

Wi-Fi signals are different in this regard. Over the last few years, millions of Wi-Fi access points have been deployed within networks by individuals, institutions and enterprises. These networks have been established to enable efficient and flexible access to data and communications. Furthermore, the strength of the signal broadcasted by Wi-Fi access points can be measured and used in algorithms to locate a Wi-Fi enabled device. Unfortunately, those signals can be affected by a diversity of uncontrollable environmental effects, such as people, building material etc., which weaken the positioning capabilities of Wi-Fi. Despite this significant limitation, Wi-Fi signals continue to be an attractive positioning technology due to the growing number of Wi-Fienabled mobile devices being produced and, combined with the rapid growth in $\mathrm{Wi}-\mathrm{Fi}$ access points being installed globally [11].

\subsection{Wi-Fi Enabled RFID}

Wi-Fi-enabled RFID offers a more accurate system than a traditional RFID network for localization of tagged objects, and is commonly used for object-tracking in a specific physical context, such as cars in a parking lot, equipment in a manufacturing plant, etc. A regular RFID system can give what is called the "choke point" location, or zone-based location, meaning the location of the tag is known only in relation to the reader detecting its presence. On the other hand, a Wi-Fi network can determine the precise $x, y$ coordinates of a tag, in the same way as GPS does, using triangulation methods [12].

In these RFID based systems, tags are Wi-Fi devices, while Wi-Fi access points function as readers. These tags are capable of bi-directional data exchange, and can determine the location within an accuracy of 3-5 meters. Furthermore, a large number of tags can concurrently communicate with a single access point, without choking the Wi-Fi network [5].

The Media Access Control (MAC) address on the tag serves as the unique identifier, as it is able of individually identifying each node of a network. The specific location coordinates are determined by specially designed location software. Additional application software can be utilised to transform coordinates into meaningful terms to the user, by matching them to specific locations. A Wi-Fi application can then identify the tag and its location. Some tags may contain additional data derived from integrated sensors [6].
Wi-Fi-enabled RFID technology has been used and proven in many applications. Kidspotter's child tracking system is an interesting example, in which Wi-Fi-enabled tags to track children in theme parks were successfully used. The system was installed at Denmark's Legoland in 2003. A traditional RFID technology would have required hundreds of readers in order to pinpoint the location of a child with the same precision as the Wi-Fi network, which required only 34 readers. This has also motivated Legoland to upgrade its existing Wi-Fi infrastructure, which it uses for point-of-sale equipment, Wi-Fi hotspots, and other functions in its theme park. The application has also been used to determine traffic patterns within the park, including where people travel and how fast or slow etc., which added more value to the services provided by the park [12].

The operation principle of Wi-Fi based active RFID tags can be described in simple terms as follows: a wireless IEEE802.11WLAN signal is transmitted by the tags at a regular interval, which is received by the $\mathrm{Wi}-\mathrm{Fi}$ access points, which in turn redirect the signal to a location device. The location device determines tag's location and sends it to the visibility software, which uses location data to determine the exact location of the RFID tag. The Wi-Fi (IEEE802.11WLAN) can support up to 128 devices within one network and can transmit data up to $30 \mathrm{~m}$, but requires more power and could incur more cost than other technologies [5]. However, the cost of the system can be justified and reduced due to the multi-functionality of the Wi-Fi network, which is indispensable within any enterprise, and is usually available beforehand. The RFID tags for the application proposed in this work can be used for this specific application for a limited time each year, which reduces the running cost of the required equipment. The economic viability of this technique should be assessed further through a more thorough economic study of required equipment, which is beyond the scope of this study [9].

\section{Localization Techniques}

Localization of the WSN tags is the most important element in matching mother and calf reindeer. Various localization techniques have been investigated and reviewed by many researchers such as Guolin et al. and Sayed et al. [13, 14], however, those researchers focused mainly on localization techniques in wireless local area network (WLAN) and cellular network environments, nevertheless, special attention was given to signal processing aspects of the localization techniques.

A review that focuses on localization algorithms and measurement techniques in WSNs was presented by Mao et al. [15]. In their paper, they explored various measurement techniques implemented in WSN localization, which included distance related measurements, Angle-Of-Arrival (AOA) measurements and Received Signal Strength (RSS) profiling techniques. Distance related measurements were classified in their work into: Received Signal Strength (RSS)-based distance measurements, Time-Difference-Of-Arrival (TDOA) 
measurements, one-way propagation time and round-trip propagation time measurements and the lighthouse approach to distance measurements. Furthermore, they explored onehop localization techniques based on these measurements and highlighted non-line-of-sight error mitigation techniques in WSN localization with focus on multi-hop localization techniques, particularly connectivity-based and distance-based multi-hop localization techniques. Open research problems in distance-based localization were also considered [15]. The two main elements of localization are range measurement and location estimation. Those will be considered more thoroughly in the following sections, based on the review presented by Bouet and Santos [9]:

\subsection{Range Measurements}

Various methods are available for range measurements. Received Signal Strength (RSS), Angle Of Arrival (AOA), Time Difference Of Arrival (TDOA), Time Of Arrival (TOA), or Received Signal Phase (RSP) are the major techniques used for this purpose. Those techniques are discussed further below:

1. RSS (Received Signal Strength): Emitted signal strength is attenuated in direct proportion to the distance between the emitter and the receiver. Hence, the corresponding signal path losses due to propagation can be used, together with at least three reference points, to localize the target. The difference between transmitted and received signal strengths have been utilized in several empirical and theoretical models for distance estimation. These systems usually need on-site adaptation in order to mitigate the negative effects of shadowing and multipath fading in indoor environments [9].

2. AOA (Angle Of Arrival): this concept involves calculation of the intersection of several direction lines originating from the target. At least two angles, measured with an array of directional antennae, and then transformed into direction lines, are required to define the 2-D location of a target. Nevertheless, complex and expensive equipment are required for this technique, and is still prone to errors arising from signal reflections and shadowing.

3. TDOA (Time Difference Of Arrival): in this technique, the times of arrival of a signal emitted by a target are measured at multiple receivers, and the difference in those values is used for determining the relative location of the targeted transmitter. Three or more receivers in $\mathrm{Wi}-\mathrm{Fi}$ access points document the arrival times of a radio signal. Subsequently, triangulation software uses the measurements to determine the transmitter's location [15]. Accurate time reference between the measuring units is very crucial for this method. TDOA suffers from downsides similar to TOA in indoor environments, where a line-of-sight (LOS) channel is rarely available [5].

4. TOA (Time Of Arrival): The distance between a reference point and the target is proportional to the signal propagation time. At least three different measuring units are required for TOA-based systems to perform a lateration for 2-D positioning. Furthermore, precise synchronization between transmitters and receivers should be achieved, in order to minimize the localization error [9].

5. RSP (Received Signal Phase): This technique, which is also known as Phase of Arrival (POA), uses the delay, expressed as a function of wavelength, to estimate the distance. Nevertheless, this technique requires a LOS signal path to reduce localization errors [9].

\subsection{Location Estimation}

In general, localization algorithms discussed in literature can be classified into three categories, namely distance based estimation, scene analysis and the probabilistic method.

\subsubsection{Distance-Based Localization}

Amongst others, two prominent location estimation techniques will be considered: triangulation, which uses the angles formed by two reference points and the target node to calculate the location of the transmitter, and trilateration where the estimated location is defined by the intersection point of three circles.

In the triangulation group of algorithms, properties of triangles are employed to estimate the target's location. The angle of incidence (or Angle of Arrival - AOA) of at least two reference points are measured, and the intersection of the two lines is used to define the estimated position of the target node, with reference to a known distance. This method is illustrated in figure 3 below [9].

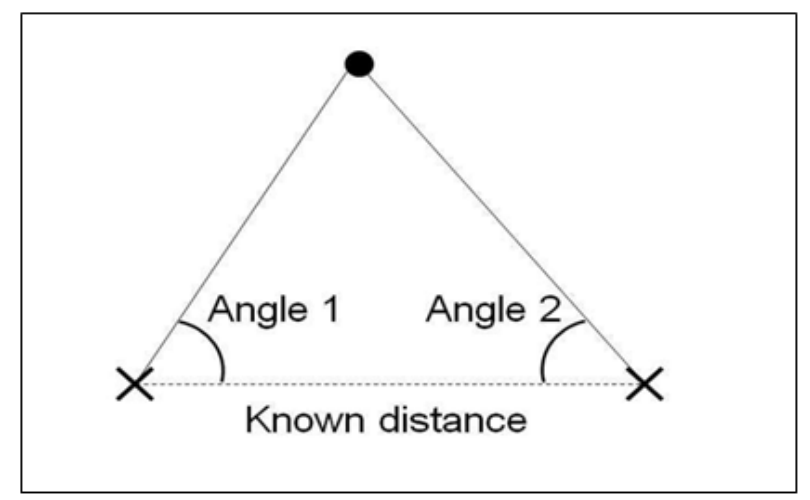

Figure 3. Triangulation: the estimated location is calculated using the angles formed by two reference points and the target node [9].

The lateration approach, on the other hand, estimates the position of the target by evaluating its distances from at least three reference points, as shown in figure 4, which shows an example of trilateration, where three reference points are used to estimate the position of the target node. Distance from the points is indicated by the radii of the circles, while the intersection point of the three circles represents the estimated location of the target point [9]. 


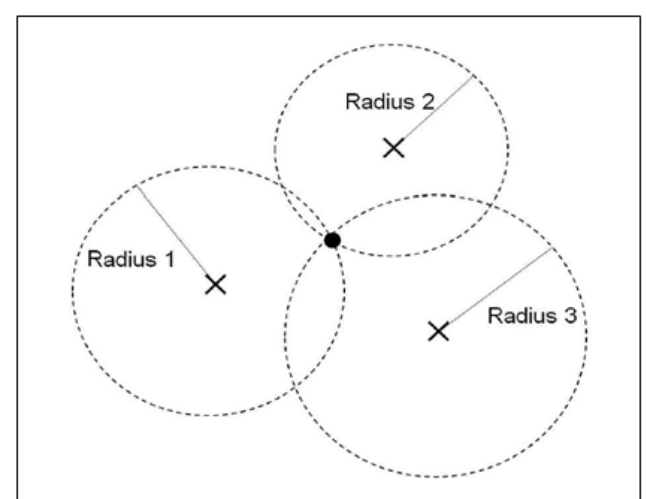

Figure 4. Trilateration: the intersection point of three circles defines the estimated location [9].

\subsubsection{Scene Analysis}

The second class of localization algorithms is the scene analysis approach, which is composed of two steps. Initially, information about the environment is gathered as (finger prints), then the target's location is estimated by matching those finger prints with online measurements. Fingerprinting is usually based on RSS. The two main fingerprinting-based techniques are: k-nearest neighbour $(\mathrm{k}-\mathrm{NN})$, where $k$ is a small positive integer, and probabilistic methods [15].

In the k-NN method, RSS measurements at known locations are gathered to build a database which is called a radio map. Those measurements are linked to the target during the online phase, and are used to search for the closest matches in the previously built signal space. [15] [9].

\subsubsection{The Probabilistic Approach}

The probabilistic approach, on the other hand, aims to find the location of a target assuming that there are $n$ possible locations and one observed signal strength vector during the online phase, according to posteriori probability and Bayes formula. This leads to the selection of the location with the highest probability. This method involves different stages such as active learning, error estimation, calibration and tracking with history [9].

\section{Conclusions}

In this paper, a novel application of WSN based on Wi-Fi enabled RFID tags is proposed for ownership identification of new-born reindeer calves, in order to mark the calves with ear marks or identification tags of their relevant owners. The principle of identification is based on matching reindeer calves to their mothers through tracking the movement of the herd, while finding a proximity between a reindeer cow and her calf.

In the proposed set-up, active RFID ear tags, which are fitted to the animals, are utilised as nodes. Such tags normally have an accuracy of 3-5 meters when determining the location, and when used with Wi-Fi gateways, it is possible to allow a large number of tags to communicate simultaneously with a single access point without choking the Wi-Fi network. This increased capacity of the system is enabled through the deployment of Wi-Fi gateways. The traditional RFID technology would have required a larger number of readers, in order to determine the location of a single tag, with the same precision as the Wi-Fi network.

The system is incomplete without the localization algorithm. Various localization algorithms and distance measurement techniques in WSNs have been considered and thoroughly discussed in this paper. Either triangulation or trilateration can be utilised to define the estimated location of the target tag, while a number of techniques are available for range measurements, which can be employed in proper computer algorithms to analyse the gathered data-base of animal tracking, among these, Time Of Arrival (TOA) seems to be the most economic and practical choice for the proposed application.

The advocated method suffers from a few limitations pertaining to the number of tags that can be covered using one Wi-Fi reader and coverage of the pen area, where the reindeer herd is gathered for identification, which could create further problems pertaining to transmission, repeatability and accuracy.

Further limitations relevant to the localization algorithms and range measurement can be attributed to the demand for complex and expensive equipment, in addition to errors arising from signal reflections and shadowing. Loss of LOS can sometimes become a challenge to certain measurement techniques and equipment, which needs to be taken into consideration. However, those limitations can be overcome using proper antennas and larger number of Wi-Fi readers.

The proposed method reduces the amount of effort and time in performing the identification process and paves the way for the implementation of Wi-Fi enabled RFID tags for animal welfare.

\section{Acknowledgements}

This work was undertaken under the Botnia-Atlantica programme, co-funded by the European Regional Development Fund.

\section{References}

[1] The International Centre for Reindeer Husbandry (ICR), "Reindeer herding," 2015. [Online]. Available: http://reindeerherding.org/about-us/. [Accessed 19 July 2015].

[2] R. Paine, "Herds of the Tundra: A portrait of Sami Reindeer Pastoralism," Smithsonian Institution Press, 1994.

[3] N. J. C. Tyler, J. M. Turi, et al, "Saami reindeer pastoralism under climate change: Applying a generalized framework for vulnerability studies to a sub-arctic social-ecological system," Global Environmental Change, vol. 17, pp. 191-206, 2007.

[4] Mustafa, M., Hansen, I. \& Eilertsen, S., "2013. Animal Sensor Networks: Animal Welfare under Arctic Conditions," in SENSORCOMM 2013: The Seventh International Conference on Sensor Technologies ans Application, Barcelona, 2013. 
[5] Mustafa, M., Hansen, I., Eilertsen, S., Pettersen, E. \& Kronen, A., "Matching Mother and Calf Reindeer Using Wireless Sensor Networks.," in 2013 5th International Conference on Computer Science and Information Technology (CSIT 2013), IEEE Conference procee, Amman, Jordan, 2013.

[6] Vishwas Raj Jain, Ravi Bagree, Aman Kumar and Prabhat Ranjan, "wildCENSE: GPS based Animal Tracking System," in ISSNIP, 2008.

[7] Kwong, K. H., T. T. Wu, et al., "Practical considerations for wireless sensor networks in cattle monitoring applications," Computer and Electronics in Agriculture, vol. 81, pp. 33-44, 2012.

[8] R. Want, "An introduction to RFID technology," IEEE Pervasive Computing, vol. 5, no. 1, pp. 25-33, 2006.

[9] Bouet, M. and A. L. d. Santos, "RFID Tags: Positioning Principles and Localization Techniques," in Wireless Days, WD '08. 1st IFIP IEEE, Dubai, 2008.

[10] Hassan, T. and S. Chatterjee, "A taxonomy for RFID," in HICSS, 2006.
[11] Kealy, A., B. Li, et al., "Evaluation of wifi technologies for indoor positioning Applications," in Surveying \& Spatial Sciences Institute Biennial International Conference, 2009.

[12] Fine, C., N. Klym, et al., "The Evolution of RFID Networks," M. Sloan School of Management, 2006. [Online]. Available: http://ebusiness.mit.edu. [Accessed 2015].

[13] Guolin, S. and C. Jie, et al., "Signal processing techniques in network-aided positioning: a survey of state-of-the-art positioning designs," IEEE Signal Processing Magazine, vol. 22 , no. 4, pp. 12-23, 2005.

[14] Sayed, A. H. and A. Tarighat, et al, "Network-based wireless location: challenges faced in developing techniques for accurate wireless location information," IEEE Signal Processing Magazine, vol. 22, no. 4, pp. 24-40, 2005.

[15] Mao, G. and B. Fidan, et al., "Wireless sensor network localization techniques," Computer Networks, vol. 51, no. 10, pp. 2529-2553, 2007.

[16] T. Rappaport, Wireless Communications: Principles and Practice, Upper Saddle River, NJ, USA: Prentice Hall PTR, 2001. 